\title{
Cavernous Hemangioma of The Testis Mimicking as Torsion of Testis: A Case Report
}

\author{
Shreekant Bharti and Narrendran A P* \\ Dept of Pathology, IMS, Banaras Hindu University, India
}

\begin{abstract}
Introduction: Cavernous hemangiomas are diagnosed by dilated large vessels with thin walls. Intra-testicular hemangiomas are a rare entity, and often mimic testicular malignancy or may present as testicular torsion.

Case Report: 18 year old male presented with a month-long history of lower abdominal pain and clinical examination revealed only a mildly enlarged, non-tender left testis. Ultrasonography of abdomen and scrotum suggested avascular hypoechoic left testis with hydro-hematocoele likely secondary to torsion of testis. A probable diagnosis of torsion of testis was made and the patient underwent left orchiectomy. Microscopic examination of the tumor revealed a cav $\neg$ ernous hemangioma composed of variably sized, clot-filled cavernous spaces separated by fibrous tissue and leading to hemorrhagic necrosis of the testicular parenchyma.
\end{abstract}

Conclusion: Clinicians need to consider the possibility of the rare but benign lesion, testicular hemangioma, in young patients presenting with testicular mass and/or pain, along with normal tumour markers and increased intralesional vascularity on imaging.

Keywords: Hemangioma, Cavernous Hemangioma, Testicular Cancer, Benign Testicular Tumor

\section{Introduction}

International Society for the Study of Vascular Anomalies (ISSVA) divided vascular anomalies into vascular tumors and vascular malformations. Vascular tumors arise mainly due to endothelial hyperplasia and they may regress spontaneously. Vascular malformations occur by localized defects of vascular morphogenesis, secondary to dysfunction in pathways regulating embryogenesis and they usually persist in adulthood ${ }^{[1]}$. In 2000, Mazal et al. reviewed the literature and identified four histopathological types of testicular hemangiomas: cavernous, histiocytoid, capillary and papillary endothelial hyperplasia ${ }^{[2]}$. In the past decade, less than 25 cases of testicular cavernous hemangioma have been reported in literature.

Cavernous hemangiomas are diagnosed by dilated large vessels with thin walls. Their walls may be abnormal which may not confirm to arterial or venous morphology. Developmental arrest during the stage of an undifferentiated capillary network has been postulated to result in a cavernous hemangioma. We report a case of an 18-year-old male patient diagnosed with hemangioma of the testis which is a rare entity.

\section{Case Report}

An 18 year old male presented with complaints of lower abdominal pain extending till left scrotum since one month. There was no history of trauma to lower abdomen or scrotum. Clinical examination demonstrated unremarkable abdominal findings with mildly enlarged, non-tender left testis and normal ipsilateral spermatic cord.

He underwent an ultrasonogram of abdomen and scrotum which revealed hypoechoic left testicular parenchyma with collection of fluid and epididymis was bulky and heterogenous. Spermatic cord showed normal vasculature. These features suggested avascular hypoechoic left testis with hydro-hematocoele likely secondary to torsion of testis. A probable diagnosis of torsion of testis was made and the patient underwent left orchiectomy.

On gross examination, the testis measured $3.5 \times 3.5 \times 1.5 \mathrm{~cm}$ with attached spermatic cord measuring $7.5 \mathrm{~cm}$ in length. The orchiectomy specimen showed a testicular tumor of $3 \times 3 \times 1.5 \mathrm{~cm}$ size which on sectioning showed blood clots, areas of necrosis and part of normal parenchyma. Microscopic examination of the tumor revealed a cavernous hemangioma composed of variably sized, clot-filled cavernous spaces separated by fibrous tissue and leading to hemorrhagic necrosis of the testicular parenchyma.

\section{Discussion}

Genital hemangiomas most likely arise from the inner layer of the tunica albuginea, which contains blood vessels and lymphatics and sends septa into the testicular parenchyma. A hemangioma may extend into the testicular parenchyma by way of these septa ${ }^{[3]}$. Intra-testicular form of hemangiomas is reported rarely, compared with extratesticular genital hemangiomas. Testicular hemangiomas 


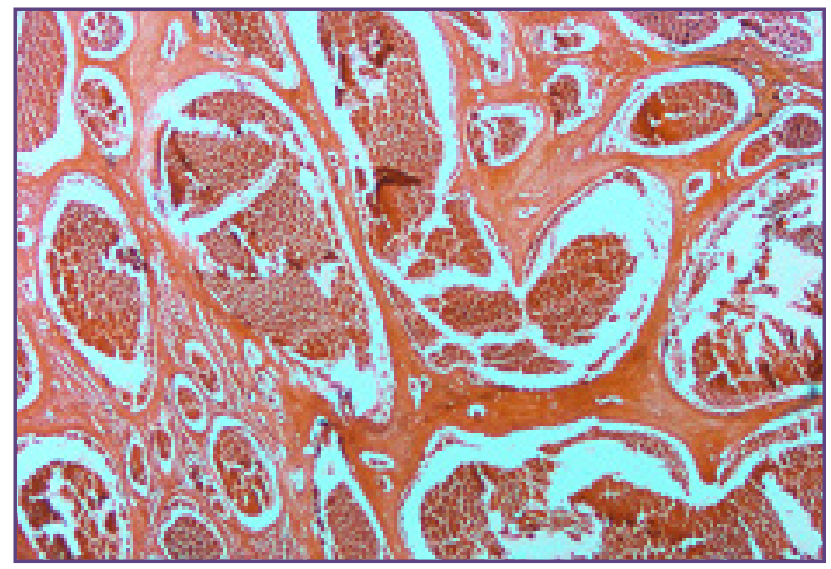

Fig. 1: Cavernous hemangioma of testis showing interconnected, thin walled blood vessels (H\&E, 40x).

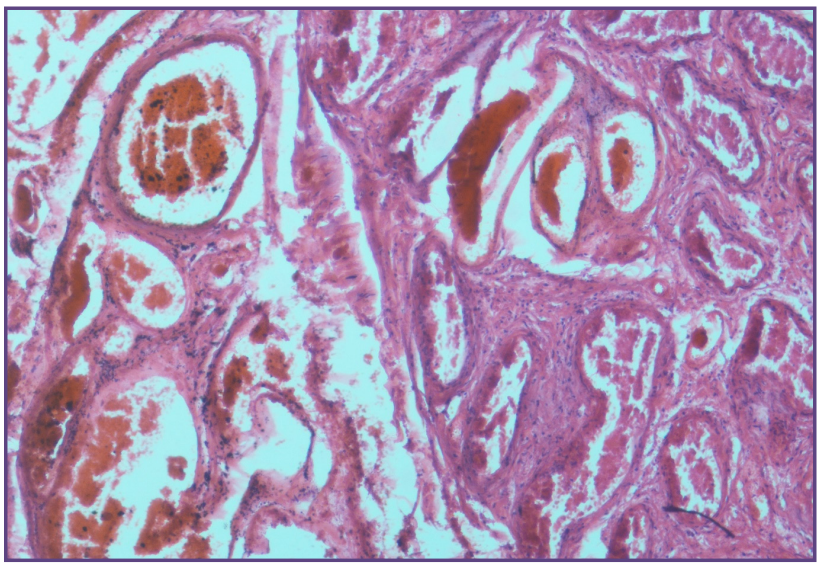

Fig. 2: Cavernous Hemangioma of testis showing endothelium lined, dilated vascular spaces separated by fibrous septae (H\&E, 100x).

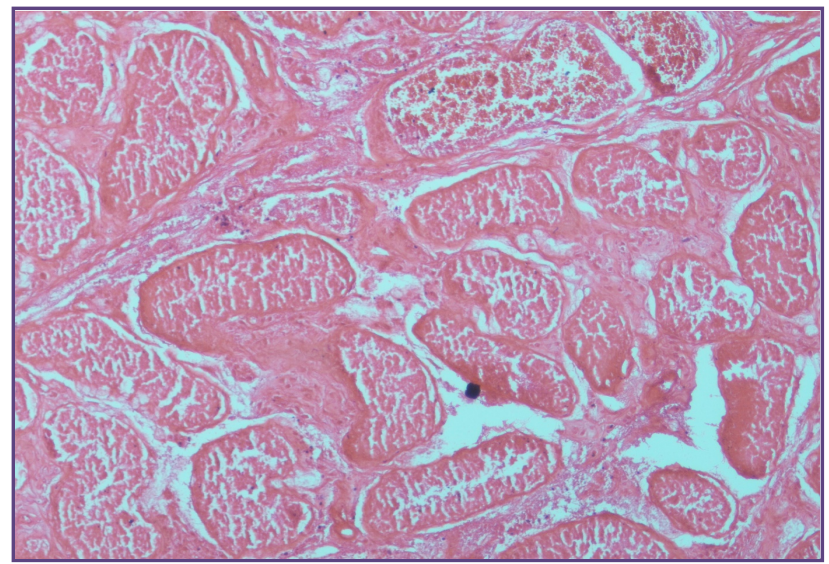

Fig. 3: Necrosis of the testicular parenchyma associated with cavernous hemangioma of testis (H\&E, 100x).

are extremely rare and they occur in diverse age groups appearing in infants to old men ${ }^{[4]}$.

Cavernous hemangioma of the testis primarily presents during childhood ${ }^{[5]}$. Unilateral testicular enlargement, with or without tenderness, is the chief presenting symptom, which is similar to that of malignant testicular tumors on clinical presentation. Testicular hemangiomas can also present as testicular torsion or associated with testicular infarction ${ }^{[6]}$. Rarely, it can present with bleeding or ulceration. Spontaneous involution is not reported with this type of hemangioma and long term complications like infertility, hemorrhage and even necrosis can occur as seen in our case. It can be seen extending into penis, anterior abdominal wall, perineum and pelvis ${ }^{[7,8]}$. An association with hemangiomas in other sites such as skin, liver, rectum and bladder has been reported by other authors ${ }^{[9,10]}$.

On ultra-sonogram, testicular hemangiomas are often hypoechoic but sometimes, hyperechoic or even heterogenous
${ }^{[11]}$. When the tumor markers are negative, presence of a mass with variably sized calcifications is highly suggestive of cavernous hemangioma. However, Serum AFP, beta-hCG and $\mathrm{LDH}$ are raised only in half of the patients diagnosed with testicular malignancies. Demonstration of intra-lesional blood flow can be made by Color Doppler studies; however lack of flow does not indicate the absence of cavernous hemangiomas. Treatment options available are surgical excision, laser fulguration, intralesional sclerotherapy and cryotherapy. Testis sparing surgeries are commonly done in pre-pubescent tumors as the proportion of benign tumors is relatively higher than adults ${ }^{[12]}$.

Apart from dilated, endothelium lined vascular spaces separated by fibrous tissue, cavernous hemangiomas also show thrombosis and calcification commonly ${ }^{[5]}$. The immunohistochemical markers CD31 and CD34 can help in delineating the endothelial lining of the cavernous spaces, while FVIII and vimentin shows variable positivity. 
Differential diagnoses of testicular cavernous hemangioma must include malignant tumors of the testis, epididymis and spermatic cord. The worldwide incidence of testicular cancer is estimated to have doubled in the last 40 years but significant variation exists between races and countries ${ }^{[13]}$. Hemangiomas and malignant tumors of the testis, especially seminomas have similar findings on ultra-sonogram and magnetic resonance imaging ${ }^{[14]}$. These findings along with the relatively high incidence of aggressive malignant tumors of testis result in patient undergoing high inguinal orchiectomy. Morphologically, the malignant tumor which forms the main differential diagnosis is well-differentiated angiosarcoma. Angiosarcoma occurs in mid and late adult life and has a broad morphologic spectrum varying from a benign hemangioma like appearance to a spindled or an epithelioid appearance. It shows infiltrative and interanastomosing growth pattern with at least focal endothelial atypia and nuclear hyperchromasia.

Genital hemangioma may mimic an inguinal hernia, while subcutaneous location on the scrotum can be confused with a varicocele when it presents on the left side. Infarction associated with torsion of testis is usually analyzed with frozen section and histopathological sections are helpful only in confirming the diagnosis. All the cases of testicular cavernous hemangioma reported in the literature have run a benign course and there were no mentions of metastasis and recurrence following surgical excision ${ }^{[15]}$.

\section{Conclusion}

Clinicians and pathologists must be aware of the rare entity of testicular hemangiomas, as clinical examination and imaging studies do not often suffice to arrive at a correct diagnosis. Testicular cancer and torsion of testis are frequent mimickers and considering their high incidence and clinical significance, diagnosis is further delayed. A young patient presenting with testicular mass and/or pain, along with normal tumour markers and increased intralesional vascularity on imaging studies should raise a suspicion of testicular hemangiomas.

\section{Acknowledgements}

We acknowledge the technical staff of the histopathology lab and the departmental head for the general support.

\section{Reference}

1. Talmon GA, Stanley SM, Lager DJ. Capillary hemangioma of the testis. Int J Surg Pathol. 2011;19:398-400.

2. Mazal PR, Kratzik C, Kain R, Susani M. Capillary haemangioma of the testis. J Clin Pathol. 2000 Aug; 53 (8):641-2.

3. G. Erdag, E. Kwon, E. Lizza, M. Shevchuk. Cavernous hemangioma of tunica albuginea testis manifesting as testicular pain. Urology. 2006 Sep;68(3):673.e13-5.

4. Numakura K, Tsuchiya N, Inoue T, Yuasa T, Matsuura S, Satoh S, Habuchi T. Case of testicular venous hemangioma. Hinyokika Kiyo. 2007 Jul;53(7):493-5.

5. Chavan D, Javalgi AP. Scrotal Hemangioma: A Case Report. Journal of Clinical and Diagnostic Research: JCDR. 2014;8(12):ND03-ND04.

6. Minagawa T, Murata Y. Testicular cavernous hemangioma associated with intrascrotal testicular torsion: a case report. Hinyokika Kiyo. 2009;55:161-163.

7. R. Rastogi. Diffuse cavernous hemangioma of the penis, scrotum, perineum, and rectum: a rare tumor. Saudi J Kidney Dis Transplant 2008;19(4):614-618.

8. Froehner M, Tsatalpas P, Wirth MP. Giant penile cavernous heman-gioma with intrapelvic extension. Urology. 1999;53:414-5.

9. Keret D, Kam I, Ben-Arieh Y, et al. Scrotal cavernous haemangioma with a family history of cutaneous angiomata. J R Soc Med. 1990;83:402-403.

10. Yanai S, Tsutsumi H, Hotsubo T, et al. Development of a testicular haemangioma after interferon therapy for hepatic haemangiomas: a case report. Eur J Pediatr. 1997;156:784-786.

11. Suriawinata A, Talerman A, Vapnek JM, et al. Hemangioma of the testis: Report of unusual occurrences of cavernous hemangioma in a fetus and capillary hemangioma in an older man. Ann Diagn Pathol. 2001;5:80-3.

12. Atkin G, Miller M, Clarkson KS, et al. Testicular capillary haemangioma in a child. J R Soc Med. 2001;94:638-40.

13. Huyghe E, Matsuda T, Thonneau P. Increasing incidence of testicular cancer worldwide: a review. J Urol 2003:170(1):5-11.

14. Takaoka E, Yamaguchi K, Tominaga T. Cavernous hemangioma of the testis: a case report and review of the literature. Hinyokika Kiyo. 2007 Jun;53(6):405-7.

15. Liu, B., Chen, J., Luo, J., Zhou, F., Wang, C., Xie, L. "Cavernous hemangioma of the testis mimicking a testicular teratoma". Experimental and Therapeutic Medicine. 2013;6.1:91-92.

*Corresponding author:

Narrendran A P, 12B, Fifth Street, North Vinayagapuram, Saravanampatti, Coimbatore - 641035. India

Phone: +91 7379141691

Email: drnarrendran@gmail.com

Financial or other Competing Interests: None.

Date of Submission : 14.10.2016

Date of Acceptance : 09.01.2017

Date of Publication : 26.03.2017 